\title{
Stability of Herbicide Resistance and GUS Expression in Transgenic Hybrid Poplars (Populus sp.) During Four Years of Field Trials and Vegetative Propagation
}

\author{
R. Meilan, D.J. Auerbach, C. Ma, S.P. DiFazio, and S.H. Strauss \\ Forest Science Department, Oregon State University, Corvallis, OR 97331-5752 \\ Additional index words. expression, herbicide tolerance, glyphosate, GUS, poplar, stability, transgene
}

\begin{abstract}
We assessed the stability of transgene expression in 40 transgenic lines (i.e., transformation events) of hybrid poplars during four years of field trials. The population consists of 40 lines of hybrid cottonwood (Populus trichocarpa $\times$ P. deltoides) grown at three field sites. All lines were transformed with a binary construct that included two genes conferring resistance to glyphosate ( $G O X$ and $C P 4)$, a gene encoding resistance to the antibiotic kanamycin $(N P T l l)$, and a visible marker gene (GUS). Agrobacterium tumefaciens was used for transformation; callogenesis and organogenesis occurred under kanamycin selection. To test the stability of transgene expression, we began repeated applications of herbicide on all lines shortly after outplanting, and challenged ramets from 40 previously untreated lines during their fourth season of vegetative growth. Here we describe how maintenance of herbicide resistance and GUS expression is indicative of transgene stability, and how we will use growth, leaf morphology, and microsatellites to investigate somaclonal variation. Preliminary data reveal that all lines that were highly resistant in year 1 continued to be highly resistant in year 4.
\end{abstract}

Since the early reports of transgene silencing by Matzke and Matzke (1991), Jorgensen (1992), and others (Finnegan and McElroy, 1994; Kooter and Mol, 1993), there has been increasing concern about the stability of transgene expression. This is especially important for trees, which undergo numerous dormancy cycles and are often exposed to extreme environmental changes during their long lives. Unstable expression has taken on greater significance given recent reports that environmental changes can trigger transgene silencing (Brandle et al., 1995; Ellis et al., 1996). Sexual reproduction can also affect the stability of transgene expression (Metzet al., 1997).

This issue is of interest to the Tree Genetic Engineering Research Cooperative (TGERC) because one of its main projects, engineering reproductive sterility, relies on stable transgene expression. The Animal and Plant Health Inspection Service (APHIS) is one of the federal agencies involved in regulating the commercialization of transgenic plants. APHIS' role with regard to agricultural biotechnology is to manage and oversee regulations to ensure the safe and rapid development of the products of biotechnology. Implicit in this statement is that the risk of transgene spread into the environment must be minimized. The easiest way to accomplish this objective is to deploy transgenic trees that are reliably sterile.

Some of our recent work has been motivated by a report from Wang et al. (1996). The authors described a population of transgenic poplars that was engineered for insect resistance, using a CrylA gene. They detected high levels of somaclonal variation in leaf morphology (Fig.1), and evidence of genetic mutation using a variety of molecular tools (i.e., random amplified polymorphic DNA, RAPD; restriction fragment length polymorphism, RFLP; amplified fragment length polymorphism, AFLP; and simple sequence repeat, SSR). Although we have observed no obvious changes in plant morphology in our transgenic populations, their findings prompted us to more carefully search our oldest field trial for similar effects. In this paper, we describe two field studies in which we investigated the stability of transgene expression and somaclonal variation. The first is a screening trial that was conducted during the 1996 and 1997 growing seasons. The primary objectives of this trial were to select lines that had commercially useful levels of resistance to glyphosate (the active ingredient in the herbicide Roundup ${ }^{\circledR}$ ) and to look at the effects of

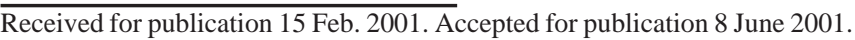

glyphosate treatment on growth. This study was continued for a second year to determine the stability of glyphosate tolerance. Before terminating the screening trial, we took hardwood (i.e.,dormant) cuttings from all untreated individuals in each transgenic line at one of the test sites. These vegetative propagules were used to establish a second study in which we continued to assess the stability of transgene expression, and to look for somaclonal variants.

\section{MATERIALS AND METHODS}

Forty independent lines (i.e., transformation events) of hybrid poplars were generated using a binary vector that contained four transcriptional units. The construct (Fig. 2) included two genes that confer resistance to glyphosate (GOX and CP4; Barry et al., 1992), a gene encoding resistance to the antibiotic kanamycin (NPTII), and the GUS reporter gene. Explants were cocultivated with a disarmed strain of Agrobacterium tumefaciens (ABI) harboring this binary vector. The transgenics were then regenerated via callogensis and organogenesis under kanamycin selection (Han et al., 2000).

The 40 lines of hybrid cottonwood (triploid P. trichocarpa Torr. \& Gray X P. deltoides Bartr. Ex Marsh) were produced in four clones (5 lines in clone 19-53, 7 in 24-305, 9 in 184-402, and 19 in 189-434). They were grown at two field sites along the Columbia River; one in western Oregon, the other was east of the Cascade Mountains. Rooted plantlets from all lines, and their corresponding nontransgenic controls, were planted in Spring 1996. Trees were treated twice with 2 and $4 \mathrm{~L} \cdot \mathrm{ha}^{-1}$ glyphosate (as Roundup Pro ${ }^{\mathrm{TM}}, 41 \%$ a.i.) during the 1996 and 1997 growing seasons. Damage due to glyphosate was assessed 4 weeks after treatment according to the damage rating system in Table1.

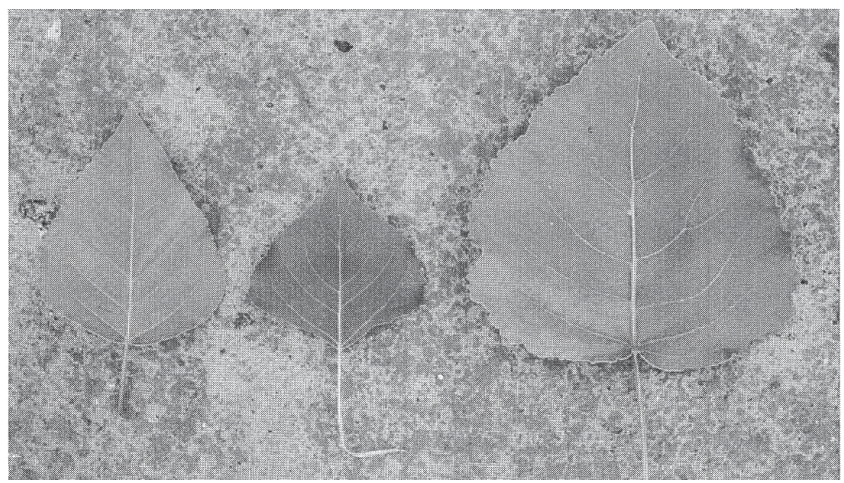

Fig. 1. Somaclonal variation in Populus nigra transgenics. These leaves show the range of morphology also described by Wang et al. (1996) in their population of insect-resistant lines.

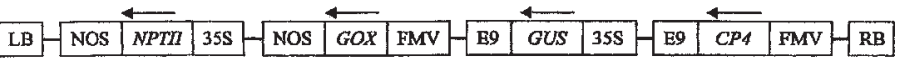

Fig. 2. Map of the T-DNA insert from the binary vector used to produce our herbicide-resistant transgenic poplars. Abbreviations: FMV, figwort mosaic virus promoter; $C P 4$, EPSPS gene from Agrobacterium tumefaciens; E9, terminator from small subunit of RUBP carboxylase from pea; 35S, 35S promoter from cauliflower mosaic virus; GUS, uidA gene from Escherichia coli; GOX, glyphosate oxidoreductase gene; NOS, nopaline synthase terminator; NPTII, neomycin phosphotransferase gene. 
Weeds were controlled manually in no-spray blocks. Growth measurements were taken during the damage estimations and again at the end of the growing season.

Hardwood cuttings were taken from unsprayed (control) lines growing in western Oregon during Winter 1997 and stored at $-3{ }^{\circ} \mathrm{C}$. In Spring 1998, these sticks were rooted in individual pots containing soil, then grown in a greenhouse until they were transplanted into the field near Corvallis, Ore. (OSU), in July 1998. At least four ramets/line were planted into each of four plots. Due to the late planting date; the short, cool growing season in 1998; and the desire to maximize uniformity of plant size, trees were coppiced in Fall 1998. They all resprouted the following spring.

To assess the stability of transgene expression this year, we sprayed two of the four plots twice during the growing season with at least 2 $\mathrm{L} \cdot \mathrm{ha}^{-1}$ glyphosate. As before, damage assessments were performed 4 weeks post-spraying. Growth measurements were taken in early June and repeated at the end of the growing season. Young leaf tissue was also sampled from each line for histochemical GUS staining. The results of this procedure will be compared with those that were obtained shortly after the transgenic plants were originally produced.

We will use a variety of approaches to investigate somaclonal variation. First, we will gather morphology data on leaves with plastochron indices of 8,9 , and 10. Leaf measurements will include overall length, petiole length, width, and distance from the base of the leaf to the point at which leaf width was determined. Collectively, these measurements will allow us to quantify changes in leaf shape. We will also evaluate shoot architecture, using methods described by Steingraeber et al. (1979). Finally, we will use our recently developed microsatellite system to search for genomic DNA rearrangements (Fig. 3).

The various treatments and conditions to which the transgenic cottonwoods were exposed are summarized in Table 2. These include many factors that have previously been shown to destabilize transgene expression. In this paper, we report on results from damage and growth measurements.

\section{RESULTS}

Screening trial. A large proportion of the transgenic lines showed complete or near-complete resistance to least glyphosate at the low spray level in 1996 ( $2 \mathrm{~L} \cdot \mathrm{ha}^{-1}$; Fig. 4). Trees with a mean damage score of 1 comprised $66 \%$ of the lines at the western site and $55 \%$ in the east. Thirty-nine transgenic lines of hybrid aspen (P. tremula L. X P. alba L., clone INRA 717-1B4) that were not a part of the stability study but were produced with the same construct and transformation method, showed a similar spectrum of resistance (OSU site). At the high spray level $\left(4 \mathrm{~L} \cdot \mathrm{ha}^{-1}\right)$, the proportions of highly tolerant lines were reduced to $48 \%, 41 \%$, and $43 \%$ at western Oregon, eastern Oregon, and OSU, respectively (data not shown).

To determine whether herbicide treatment affected growth of the transgenic trees, we calculated relative growth for each line. This was necessary because trees varied widely in size when originally planted out. For each tree, volume index was calculated as: [(basal diameter) ${ }^{2}$ $\times$ (height)]. Growth was estimated by subtracting the volume index at the beginning of the study from the volume index 1 month after the final treatment. Relative growth was defined as the log of growth for untreated trees subtracted from the log of growth for the corresponding treated line $[\log ($ trt. growth $)-\log ($ untrt. growth $)=\log ($ trt. growth $/$ untrt. growth)]. For example, trees with a relative growth value of " 1 " grew 10 times faster when treated with glyphosate than did their untreated counterparts. Approximately 50\% of the lines were unaffected by treatment (relative growth value of "0"), and about $25 \%$ of the lines appeared to grow faster when sprayed with glyphosate (Fig. 5). A similar pattern was seen for plants receiving the high rate (data not shown).

Expression study. A paired $t$ test revealed that the effect of spraying on growth was significantly lower in the 1999 expression study than it was in the 1996 screening trial. This finding is demonstrated graphically in Fig. 6, where relative growth was calculated as: $\log _{\mathrm{e}}$ (growth of unsprayed plants/growth of sprayed plants). Thus, a more negative number implies that the sprayed trees performed better
Table 1. Rating system used to score herbicide damage on the transgenic cottonwoods.

\begin{tabular}{ll}
\hline \hline Score & \multicolumn{1}{c}{ Description } \\
\hline 0 & No visible damage \\
1 & $1 \%$ to $20 \%$ of total leaf area is chlorotic \\
2 & $21 \%$ to $40 \%$ of total leaf area is chlorotic \\
3 & $41 \%$ to $60 \%$ of total leaf area is chlorotic \\
4 & 61\% to $80 \%$ of total leaf area is chlorotic \\
5 & 81\% to 100\% of total leaf area is chlorotic \\
6 & Dead \\
\hline
\end{tabular}

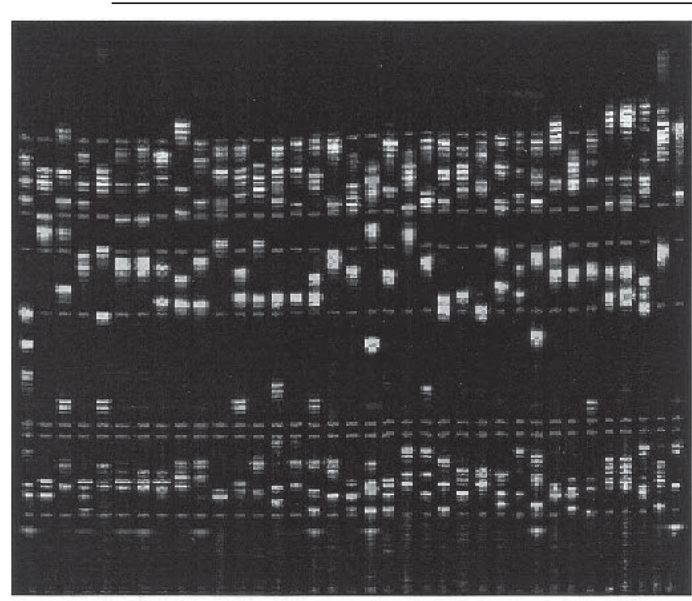

Locus 1 Locus 2 Locus 3

Fig. 3. Image of a microsatellite gel. Five fluorometrically labelled SSR primer pairs were used to amplify separate loci contained in DNA isolated from wild trees growing in eastern Oregon. Two pairs of primers were both labeled with green and blue dyes but did not amplify products that overlapped in size. The red bands are size standards that were incorporated into the PCR mixture prior to loading on the polyacrylamide gel.

Table 2. History of the transgenic cottonwood lines analyzed for stability of herbicide resistance.

\begin{tabular}{ll}
\hline \hline Event & \multicolumn{1}{c}{ Year } \\
\hline Transformants produced & $1994-95$ \\
Propagation for screening trial & 1995 (winter) \\
Propagules planted out for 2-year screening trial & 1996 (spring) \\
Hardwood cuttings taken & 1997 (winter) \\
Hardwood cuttings rooted in a greenhouse & 1998 (spring) \\
Rooted cuttings planted in field & 1998 (summer) \\
Field-grown plants coppiced & 1998 (winter) \\
Herbicide treatments begun & 1999 (spring) \\
\hline
\end{tabular}

than their corresponding unsprayed controls. If plants performed equally well in both trials, all points would lie on the diagonal line. The existence of points to the left of the diagonal indicates that treated plants grew better in 1999 than in 1996.

Despite their better growth in 1999, these trees exhibited more damage (Fig. 7). This explains the preponderance of points to the right of the diagonal, particularly near the origin $(0,0)$. Two points fell on the origin itself. Higher damage in 1999 is also evident from the relationship between relative growth and mean damage for the 2 years (Fig. 8). Most of the points on the right-hand side of this scatter plot are from the 1999 trial.

In addition to the good correlation between damage seen in 1996 and in 1999 (Fig. 7), the trees exhibiting the lowest level of damage in the screening trial appeared to have the lowest level of damage in the expression study. To test for this, all lines were ranked with respect to mean damage. A Kendall-Tau analysis revealed that the rankings for the 2 years were not significantly different. As a result, the correlation between rankings for the 2 years was highly significant (Fig. 9).

\section{DISCUSSION}

Significance of results. With a moderate transformation effort, it is possible to produce numerous transgenic lines that have commercially useful levels of glyphosate resistance. For most of the resistant lines, sprayed and unsprayed plants grew equally. The growth benefit we 


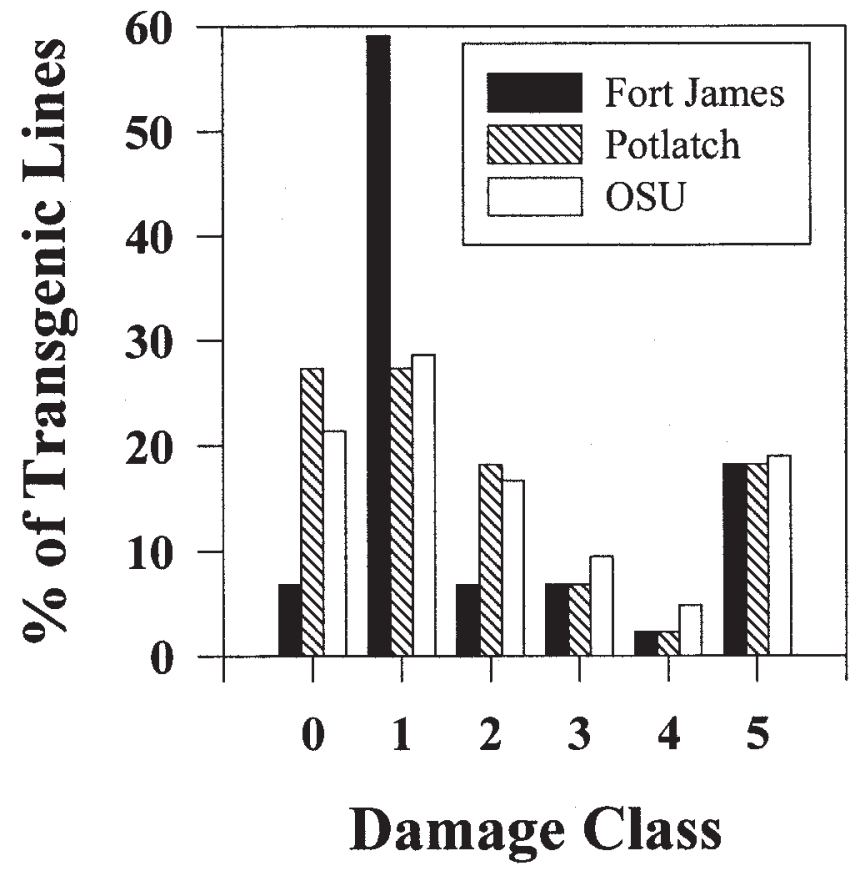

Fig. 4. Mean damage to transgenic poplars 4 weeks after the first glyphosate treatments in 1996 and 1997 (rate of $2 \mathrm{~L} \cdot \mathrm{ha}^{-1}$ ). Damage ratings (as defined in Table 1) are based on means of all ramets within each line. There were 40 lines of transgenic cottonwood at the west and east Oregon sites, and 39 lines of transgenic aspen at the OSU site.

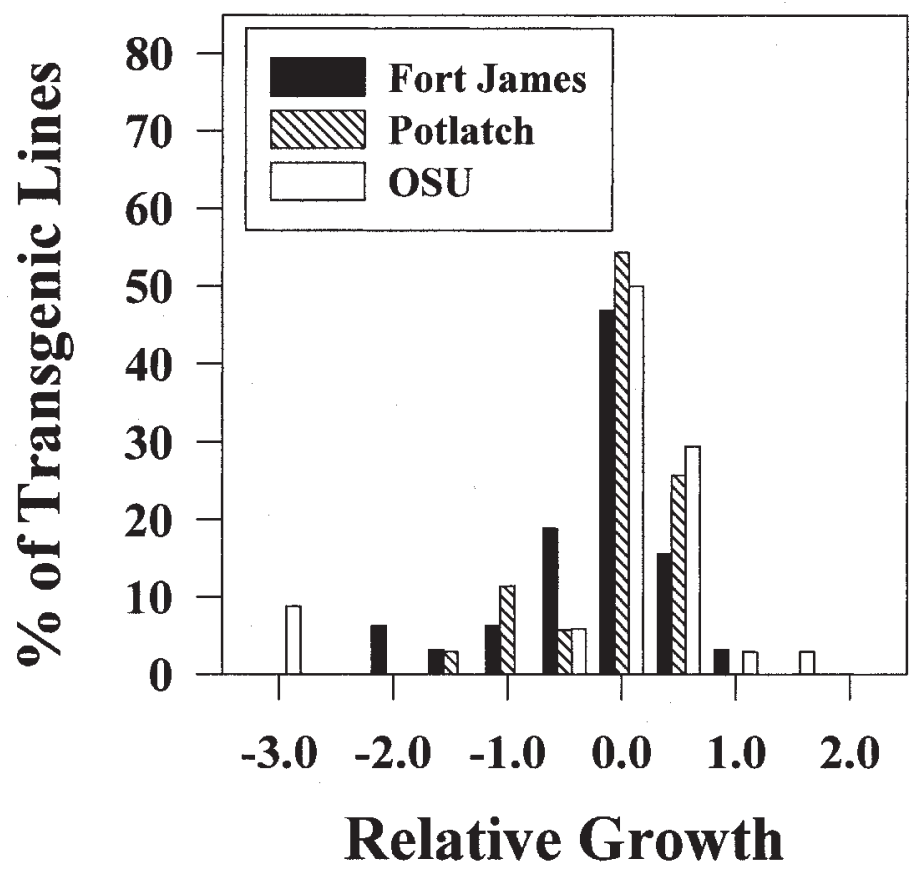

Fig. 5. Effects of glyphosate on relative growth of transgenic lines. Volume = diameter ${ }^{2} \times$ height $(\mathrm{cm})$; growth $=($ ending vol. - starting vol. $)$; and relative growth $=\{\log ($ trt. growth/non-trt. growth) $\}$. "Starting" is the time of the first treatment in 1996 and "ending" is 4 weeks after the second spraying in 1997.

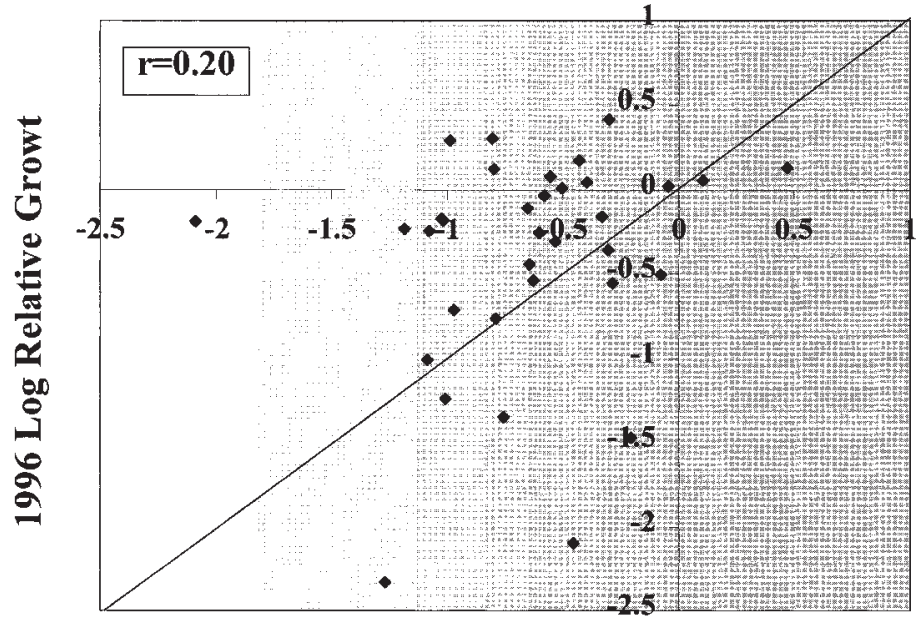

1999 Log Relative Growth

Fig. 6. The relationship between relative growth in 1996 and 1999. The transgenic lines sprayed with $2 \mathrm{~L} \cdot \mathrm{ha}^{-1}$ glyphosate in the screening trial were the same lines receiving treatment in the expression study.

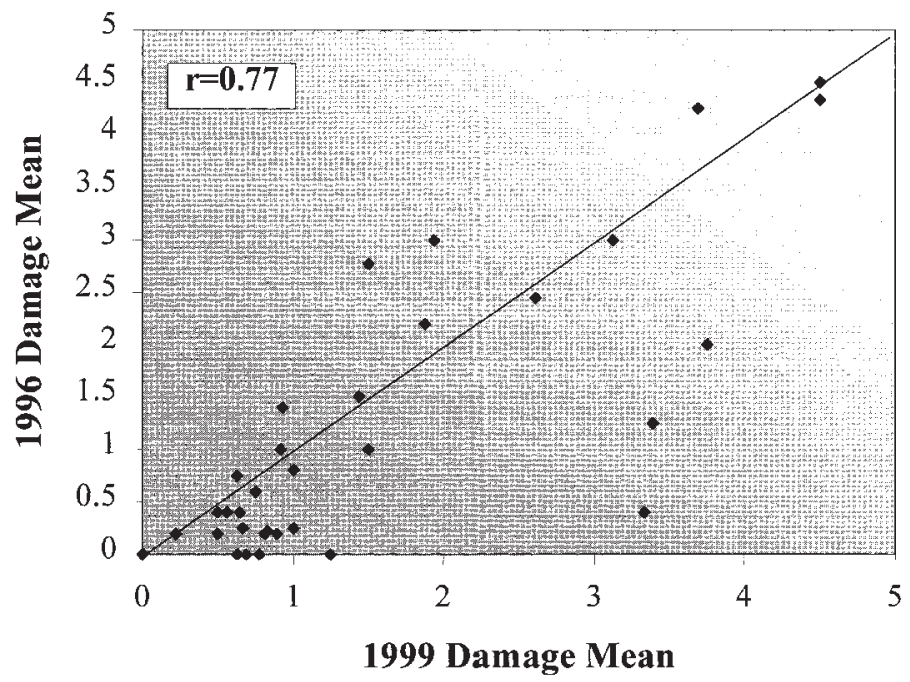

Fig. 7. The correlation between mean damage ratings assigned in the 1996 screening trial and the 1999 expression study. The trees in the screening trial received $2 \mathrm{~L} \cdot \mathrm{ha}^{-1}$ glyphosate.

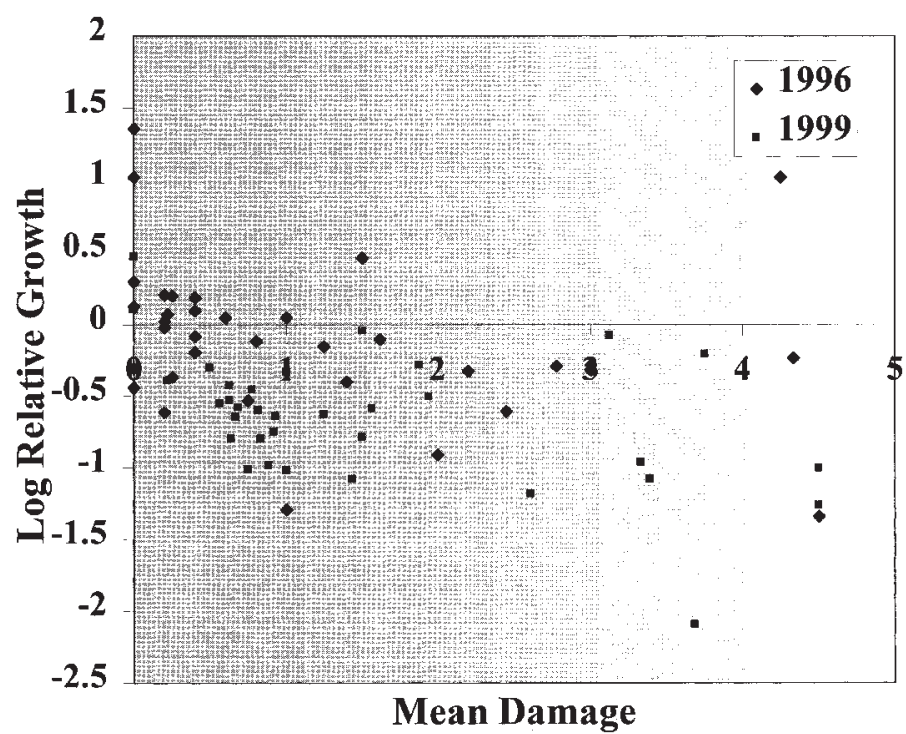

Fig. 8. Relative growth vs. herbicide damage for both the 1996 screening trial (low rate) and the 1999 expression study. 


\section{Colloquium}

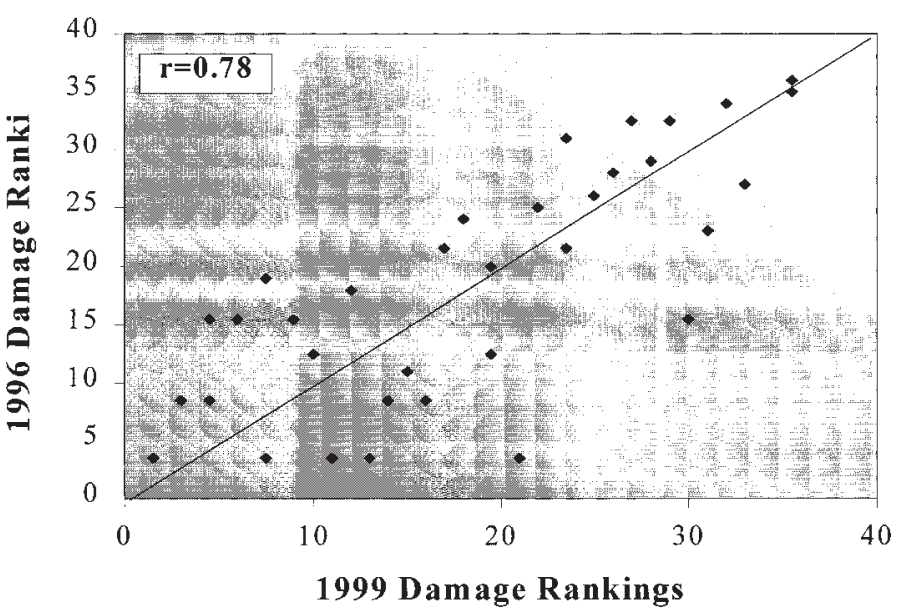

Fig. 9. The relationship between herbicide damage rankings in 1996 screening trial and 1999 expression study.

saw (treated transgenics that grew better than their corresponding unsprayed controls) was probably an artifact of differential weed control. This may not apply to operational plantations.

The greater absolute damage seen in 1999 is mostly likely due to excessive herbicide. In the 1999 trial, glyphosate was mistakenly applied at a rate of $5 \mathrm{~L} \cdot \mathrm{ha}^{-1}$. This was higher than both rates used in 1996-97, and was higher than we had originally planned to use. However, the correlation between damage in 1996 and 1999 and the similar damage rankings indicate that the transgenics displayed the same relative level of resistance in the 2 years. Thus, plants that showed little or no damage in response to the highest level of glyphosate in 1996 also showed little or no damage in 1999. This suggests that transgene expression is stable under field conditions even after vegetative propagation.

The upper two of the three outliers shown in the lower right quadrant of Fig. 7 are of low concern to us. The initial damage scores were sufficiently high that the trees they represent would not have been selected for further use. However, the third point represents a tree that went from a damage score of 0.4 in the selection trial to 3.3 in the stability study. This tree will need be investigated more fully to explain the loss of resistance. It is reassuring that only one line of the 40 tested had inadequate transgene stability.

Other factors that can influence the stability of transgene expression include in vitro culture conditions, promoter type, number of TDNA inserts, and physiological status of the plant (reviewed in Ahuja, 1997). Careful consideration must be given to all of these variables when designing transgene stability studies. The results from our stability study are consistent with those reported by Ellis et al. (1996). They showed that the highest and least variable transgene expression occurred in vitro, when compared with greenhouse- or field-grown plants. However, once a line was selected in the field, it tended to exhibit very little year-to-year variation in transgene expression. In addition, the researchers observed that genotype, season of the year, and tissue type influenced expression. We have also observed consistent expression in 53 lines engineered for insect resistance, using a toxin gene from Bacillus thuringiensis, and grown in the field for 2years (unpublished data). Thus, stability of transgene expression seems to be a minor concern for poplars, so far.

Future research. Tissue has been collected for histochemical GUS staining. Genomic DNA has been extracted from all transgenic lines and will soon be subjected to polymerase chain reaction (PCR) using five pairs of fluorometrically labeled, simple sequence repeat (SSR) primers. Leaf and crown morphology data also will be gathered shortly.

The elite lines selected in our screening trial were propagated to establish stool beds. Sticks from these stool beds were used to install multiple-acre management trials east and west of the Cascade Mountains in Spring 1999. We plan to grow the trees in these trials for a full economic rotation (8 to 10 years) to assess operational-scale stability and to compare the efficacy of conventional weed-control regimes with those involving the exclusive use of glyphosate. This will be the ultimate test of stability and will help us determine the value of engineered herbicide resistance for weed control.

\section{Literature Cited}

Ahuja, M.R. 1997. Transgenes and genetic instability, p. 90-100. In: N.B. Klopfenstein, Y.W. Chun, M.-S. Kim, and M.R. Ahuja (eds.). Micropropagation, genetic engineering, and molecular biology of Populus. Gen. Tech. Rpt. RM-GTR-297, USDA, For. Serv., Rocky Mountain For. and Range Expt. Sta., Fort Collins, Colo.

Barry, G., G. Kishore, S. Padgette, M. Taylor, K. Kolacz, M. Weldon, D. Re, D. Eichholtz, K.Finche, and L. Hallas. 1992. Inhibitors of amino acid biosynthesis: Strategies for imparting glyphosate tolerance to crop plants, p. 139-145. In: B.K. Singh, H.E. Flores, and J.C. Shannon (eds.). Biosynthesis and molecular regulation of amino acids in plants. Amer. Soc. of Plant Physiologists, Rockville, Md.

Brandle, J.E., S.G. McHugh, H. Labbe, and B.L. Miki. 1995. Instability of transgene expression in field grown tobacco carrying the $c s r l-1$ gene for sulfonylurea herbicide resistance. Biol. Technol. 13:994-998.

Ellis, D.D., J. Rintamaki-Strait, K. Francis, K. Kleiner, K. Rafa, and B. McCown. 1996. Transgene expression in spruce and poplar: From the lab to the field, p. 159-163. In: M.R. Ahuja, W. Boerjan, and D.B. Neale (eds.). Somatic cell genetics and molecular genetics of trees. Kluwer Academic Publishers, Dordrecht, The Netherlands.

Finnegan, J. and D. McElroy. 1994. Transgene inactivation: Plants fight back! Biol. Technol. 11:883-887.

Han, K.-H., R. Meilan, C. Ma, and S.H. Strauss. 2000. An Agrobacterium transformation protocol effective in a variety of cottonwood hybrids (genus Populus). Plant Cell Rpt. 19:315-320.

Jorgensen, R. 1992. Silencing plant genes by homologous transgenes. Agritech News Information 4:265N-273N.

Kooter, J.M. and J.N.M. Mol. 1993. Trans-inactivation of gene expression in plants. Curr. Opin. Biotechnol. 4:166-171.

Matzke, M.A. and A.J.M. Matzke. 1991. Differential inactivation of a transgene in plants by two suppressor loci containing homologous sequences. Plant Mol. Biol. 16:821-830.

Metz, P.L.J., E. Jacobsen, and W.J. Stiekema. 1997. Occasional loss of expression of phosphinothricin tolerance in sexual offspring of transgenic oilseed rape (Brassica napus L.). Euphytica 98(3):189-196.

Steingraeber, D.A., L.J. Kascht, and D.H. Franck. 1979. Variation of shoot morphology and bifurcation ratio in sugar maple (Acer saccharum) saplings. Amer. J. Bot. 66(4):441-445.

Wang, G., S. Castiglione, Y. Chen, L. Li, Y. Han, Y. Tian, D.W. Gabriel, Y. Han, K. Mang, and F. Sala. 1996. Poplar (Populus nigra L.) plants transformed with a Bacillus thuringiensis toxin gene: Insecticidal activity and genomic analysis. Transgenic Res. 5:289-301. 